\title{
Cygnus: development of a high resolution TPC for rare events
}

\author{
Davide Pinci*, Emanuele Di Marco, Francesco Renga and Cecilia Voena \\ INFN - Sezione di Roma, Italy \\ E-mail: davide.pinci@romal.infn.it, emanuele.dimarco@roma1.infn.it, \\ francesco.renga@romal.infn.it, cecilia.voena@romal.infn.it
}

\author{
Elisabetta Baracchini, Giovanni Mazzitelli and Alessandro Tomassini \\ INFN - Laboratori Nazionali di Frascati, Italy \\ E-mail: elisabetta.baracchini@lnf.infn.it, \\ giovanni.mazzitelli@lnf.infn.it, alessandro.tomassini@lnf.infn.it
}

\section{Gianluca Cavoto, Vasile Cristian Antochi}

Dipartimento di Fisica della Sapienza, Università di Roma, Italy

E-mail: gianluca.cavoto@phys.uniromal.it

Michela Marafini

Museo Storico della Fisica e Centro Studi e Ricerche E. Fermi, Roma, Italy

E-mail: michela.marafinieromal.infn.it

The R\&D of a gas detector prototype for high precision tracking of low energy nuclear recoils over large volume is presented in this paper. The scintillation light accompanying the electronic avalanches in a triple GEM structure is detected by a CMOS-based camera. The sensor provides very high granularity along with a very low noise and high sensitivity. Space resolution of the order of tens of $\mu \mathrm{m}$ were measured on the GEM plane $(x y)$. The Negative Ion Drift method to reconstruct the depth of track within the sensitive volume was studied. Recent tests on beam demonstrated the feasibility of the NID method with a small amount of $\mathrm{SF}_{6}$ even at nearly ambient pressure. The use of a concurrent light readout by means of a suitable photomultiplier provides the necessary timing informations. A time resolution of the order of 5 ns was measured.

EPS-HEP 2017, European Physical Society conference on High Energy Physics

5-12 July 2017

Venice, Italy

\footnotetext{
* Speaker.
} 


\section{Introduction}

The CYGNUS collaboration is developing different technologies to build an underground multi-site 'ton' gas target, for nuclear recoil measurements with a TPC readout. The final technology has to fulfill two important requirements:

1. Directionality. 3D reconstruction of tracks to exploit asymmetries and direction modulation of the Dark Matter signals for background discrimination;

2. Sensitive Volume Fiducialization. Measurement of the event position within the detector to reject events close the detector walls, very likely due to decays of radioactive materials.

The Time Projection method is an ideal candidate to achieve these results. Large volumes can be readout by means of a moderate number of channels providing a complete $3 \mathrm{D}$ reconstruction of the charged tracks within the sensitive volume. It allows the measurement not only of the total released energy but also of the energy release density along the tracks that can be very useful for particle identification and to solve the head-tail ambiguity of the tracks. Moreover, gas represents a very interesting target to study Dark Matter interactions. In gas, nuclear recoils can travel enough to give rise to tracks long enough to be acquired and reconstructed. Finally, TPC might even include light anisotropic targets with an additional directional sensitivity as suggested in [1] and [2].

For equipping large surfaces, the use of Micro Pattern Gas Detectors is a very simple solution ensuring high space and time resolution. In particular Gas Electron Multipliers [3] are able to suppres the Ion Back Flow inside the sensitive volume.

Time Projection Chambers for Dark Matter search have a crucial difference with those used in High Energy Physics experiments. With penetrating tracks an external trigger can be exploited to measure the electron drift time to evaluate the event depth. In DM applications an alternative technique is needed. One possibility the is given by the Negative Ion Drift (NID) method [4]. If a small amount of a highly electronegative component $\left(\mathrm{SF}_{6}, \mathrm{CS}_{2}\right)$ is present in the gas mixture, ionization electrons can be absorbed its molecules creating negative ions. These will start drifting in the electric fields, following the field lines with neglegible diffusion. At the multiplication stage, exceeding electrons are released and avalanches develop. If different electronegative species are present, with different masses and mobilities, they will drift with different velocities.

The measurement of the difference of the time of arrival will allow to evaluate the depth of the event inside the gas volume.

\section{Negative Ion Drift Measurements}

Negative ion drift was tested with NITEC prototype [5], a $5 \mathrm{~cm}$ gas gap readout by a $3 \times 3 \mathrm{~cm}^{2}$ triple GEM structure. The time and charge of signals induced by the electrons produced in the last GEM are acquired by means of a TimePix [6] NID technics was recorded with several different $\mathrm{CF}_{6}$-based gas mixtures. and drift velocities of the order of $\mathrm{cm} / \mathrm{ms}$, as expected for ion drifts, were measured and are shown on the left in Fig. 1 as a function of the drift electric field. The main result was the detection of the NID at nearly atmospheric pressure (610 Torr) in a mixture $\mathrm{He} / \mathrm{CF}_{4} / \mathrm{SF}_{6}$ 59.0/39.4/1.6. 

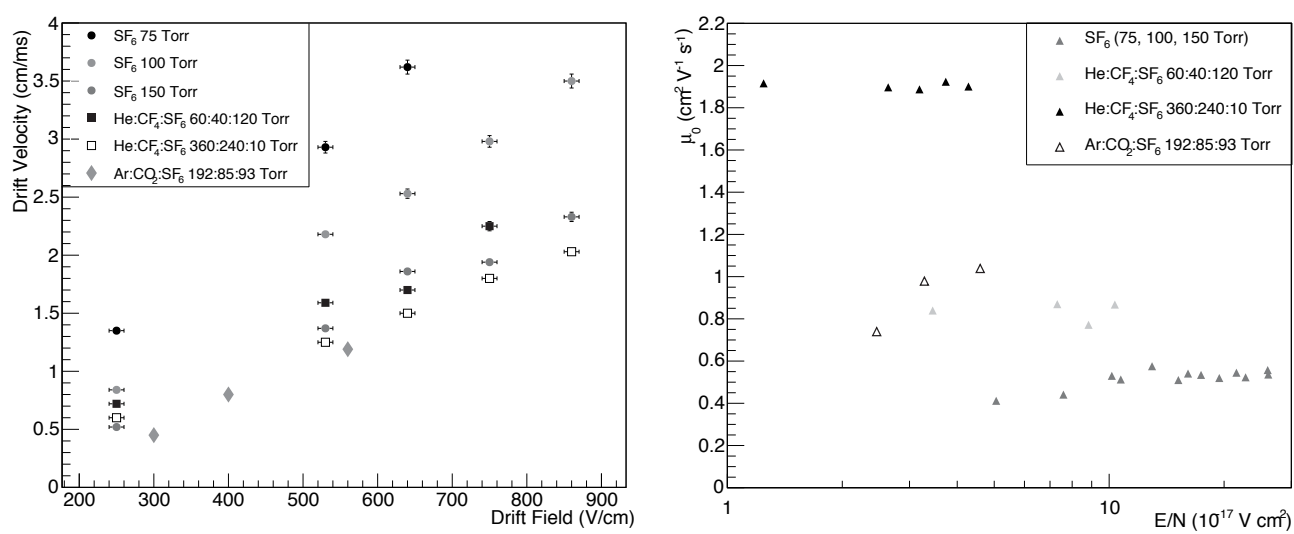

Figure 1: Negative ion velocities and mobilities as a function of the reduced electric field.

On the right of Fig. 1 the measured negative ion mobilities are shown as a function of the reduced electric field. Data acquired at 610 Torr allowed to measure the ion mobilitiy around $1.2 \times 10^{17} \mathrm{~V} \mathrm{~cm}$.

\section{Reading the light: a change of paradigm}

During the multiplication processes, photons are produced along with electrons by the gas through atomic and molecular de-excitation.

Optical readout of the light produced by GEM based detectors was already studied in the past ([8], [9]) and showed several advantages:

- optical sensors offer higher granularities with respect to electron sensitive devices;

- optical coupling allows to keep sensor out of the sensitive volume reducing the interference with high voltage operation and lowering the gas contamination;

- the use of suitable lens allow to acquire large surfaces with small sensors;

Moreover, in last years, optical sensors had a huge development and are able to provide larger granularities along with very low noise level and high sensitivity.

\subsection{Measurements of the optical readout perfomance}

The optical readout approach was tested on electron beam at the "Beam Test Facility" [7] by using the ORANGE (Optically ReAdout GEm) prototype ([10], [11]) . A $10 \times 10 \mathrm{~cm}^{2}$ Triple GEM structure, with a $1 \mathrm{~cm}$ high drift gap, gas flushed with an $\mathrm{He} / \mathrm{CF}_{4} 60 / 40$ mixture was readout by an Orca Flash 4 CMOS-based camera ${ }^{1}$ equipped with a large aperture (f/0.95) lens.

Very clear images of high energy electrons from beam and low energy electrons due to natural radiactivity, as the ones shown respectively on the left and right of Fig. 2, were acquired by this device.

\footnotetext{
${ }^{1}$ For more details visit the site www.hamamatsu.com
} 

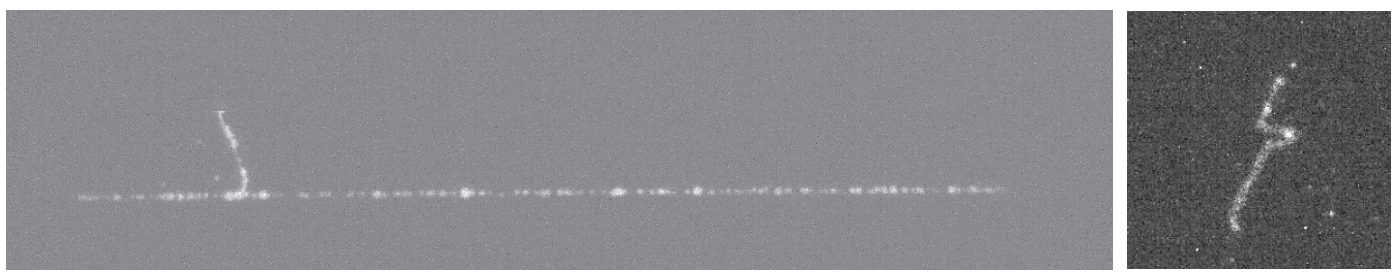

Figure 2: Examples of acquired images: on the left a $450 \mathrm{MeV}$ electron with a $\delta$ ray and on the right a low energy electron from natural radioactivity.

\subsubsection{Tracking performance and energy resolution}

The performance of the optical readout method were studied by analysing the tracks acquired by the CMOS sensor. About 1000 photons are collected per track mm, i.e. around 150 per primary electron. A so large amount of information allows to reconstruct very precisely the track position resulting in a space resolution of $35 \mu \mathrm{m}$ as shown on the left in Fig.3.
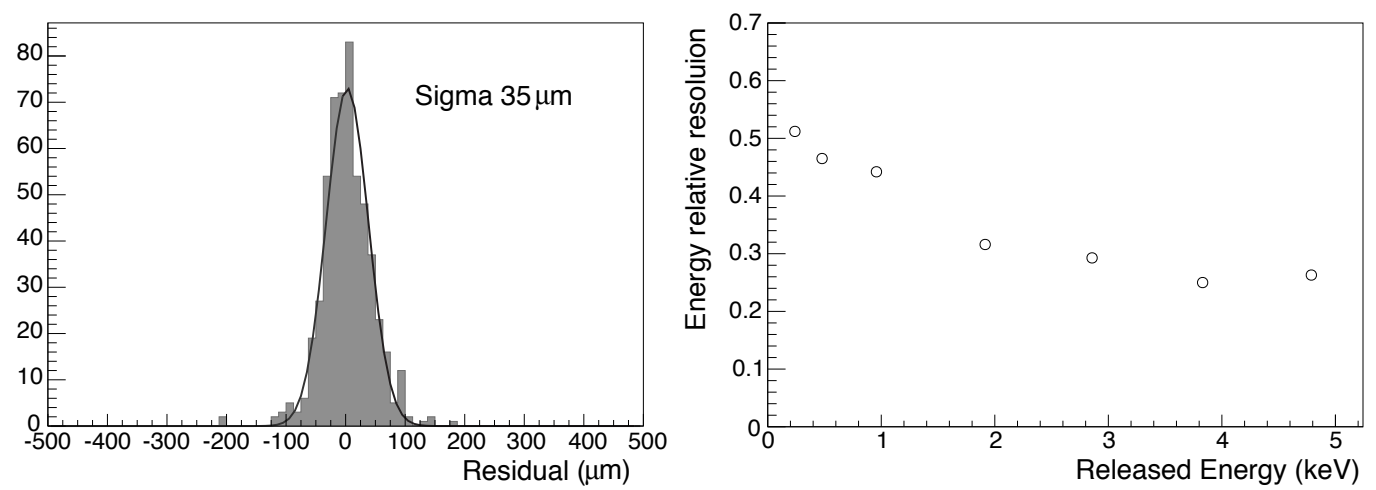

Figure 3: Left: distribution of the residuals of the reconstructed clusters to the fitted tracks with a superimposed gaussian fit. Right: relative energy resolution as a fuction of the energy released in the gas.

By studying the distribution of the light collected in slices of different widths, the energy resolution behavior was evaluated.

Figure 3 shows on the right that, already for energy realeases around $1 \mathrm{keV}$, a resolution of about $50 \%$ is obtained and that for releases in the range $3 \div 5 \mathrm{keV}$ an energy resolution better than $30 \%$ can be achieved.

\subsubsection{Exploting the time information: combined readout}

In order to acquire the time structure of the signals, light was concurrently readout by a $\mathrm{PMT}^{2}$. The waveforms of the PMT output and of the electric signal induced by the motion of the electrons on the third GEM bottom electrode (G3D) were acquired by means of a $10 \mathrm{GS} / \mathrm{s}$ sampling oscillo-

${ }^{2}$ Hamamatsu H10580 
scope $^{3}$. These two signals are able to provide, not only the time structure of the event, but also two independent measurements of the total released energy.

In Fig. 4 examples of acquired data are shown. On the left, the PMT output and the signal induced on the bottom electrode of the third GEM in a event of a single electron traveling in the drift gap, parallel to the GEM foils are shown. In both cases clear and narrow (less than 10 ns FWHM) waveforms are visibile. The relative noise level in the electric signal is considerably larger, most likely due to jitter on the high voltage supply line.
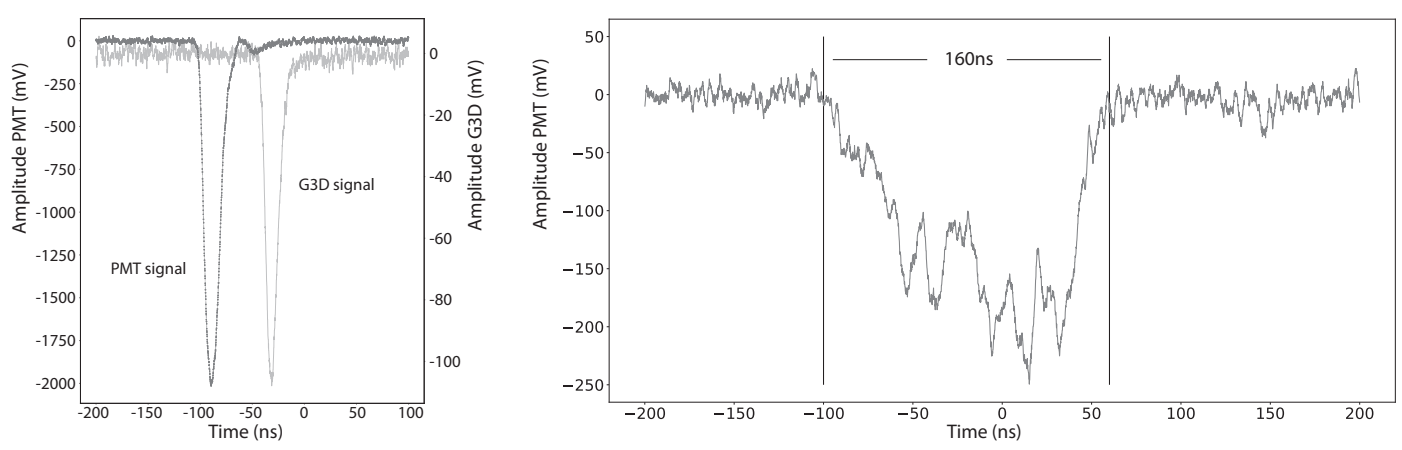

Figure 4: Example of the acquired waveforms of the PMT output and the electric signal induced on the bottom electrode of the third GEM (G3D).

From a gaussian fit of the PMT output, a sigma of $5.5 \mathrm{~ns}$ was measured. Given an electron drift velocity (calculated with Garfield [12]) of $74 \mu \mathrm{m} / \mathrm{ns}$, a resolution on the $z$ coordinate of 400 $\mu \mathrm{m}$ was evaluated.

On the right of Fig. 4, the PMT signal is shown for an inclined electron crossing the $1 \mathrm{~cm}$ drift gap at an angle of $30^{\circ}$ with respect to the GEM foils. The arrival time of the larger clusters is clearly visible, allowing an independent reconstruction of their absolute position in $z$. Taking into account the gap height $(1 \mathrm{~cm}$ ) and the width of the signal (about $160 \mathrm{~ns}$ ), an electron drift velocity of $62 \mu \mathrm{m} / \mathrm{ns}$ in agreement with the value evaluated with Garfield.

\section{Conclusion}

With the aim of constructing a high resolution GEM-based Time Projection Chamber for the study of rare processes, several R\&D activities are being carried on. On one hand, the feasibility of using Negative Ion Drift at nearly atmospheric pressure adding a small amount of $\mathrm{SF}_{6}$ to a $\mathrm{He} / \mathrm{CF}_{4}$ (60/40) gas mixture was demonstred. On the other hand, the performance of a combined optical readout (CMOS+PMT) was studied in detail. Space resolutions of $35 \mu \mathrm{m}$ on the GEM plane and of $400 \mu \mathrm{m}$ on the orthogonal direction were measured. Energy resolutions better than $30 \%$ have been achieved for energy releases in the range $3 \div 5 \mathrm{keV}$. These results make a GEM-based TPC optically readout a very promising and sensitive detector for rare process and Dark Matter search applications.

\footnotetext{
${ }^{3}$ Lecroy Waverunner 7300
} 


\section{References}

[1] G. Cavoto, E. N. M. Cirillo, F. Cocina, J. Ferretti and A. D. Polosa, "WIMP detection and slow ion dynamics in carbon nanotube arrays,” Eur. Phys. J. C 76 (2016) no.6, 349 doi:10.1140/epjc/s10052-016-4193-7

[2] L. M. Capparelli, G. Cavoto, D. Mazzilli and A. D. Polosa, "Directional Dark Matter Searches with Carbon Nanotubes,” Phys. Dark Univ. 9-10 (2015) 24 Erratum: [Phys. Dark Univ. 11 (2016) 79] doi:10.1016/j.dark.2015.12.004, 10.1016/j.dark.2015.08.002

[3] F. Sauli, "GEM: A new concept for electron amplification in gas detectors," Nucl. Instrum. Meth. A 386 (1997) 531. doi:10.1016/S0168-9002(96)01172-2

[4] C. J. Martoff, D. P. Snowden-Ifft, T. Ohnuki, N. Spooner and M. Lehner, "Suppressing drift chamber diffusion without magnetic field," Nucl. Instrum. Meth. A 440 (2000) 355. doi:10.1016/S0168-9002(99)00955-9

[5] E. Baracchini, G. Cavoto, G. Mazzitelli, F. Murtas, F. Renga, S. Tomassini, âĂIJNegative ion Time Projection Chamber operation with $\mathrm{SF}_{6}$ at nearly atmospheric pressureâĂI arXiv:1710.01994 [physics.ins-det]

[6] X.Llopart, R.Ballabriga, M.Campbell, L.Tlustos, W.Wong, “Timepix, a 65k programmable pixel readout chip for arrival time, energy and/or photon counting measurements" Nucl. Instrum. Meth. A 581 (2007) 485. doi.org/10.1016/j.nima.2007.08.079

[7] A. Ghigo, G. Mazzitelli, F. Sannibale, P. Valente and G. Vignola, "Commissioning of the DAFNE beam test facility,” Nucl. Instrum. Meth. A 515 (2003) 524. doi:10.1016/j.nima.2003.07.017

[8] L. M. S. Margato, F. A. F. Fraga, S. T. G. Fetal, M. M. F. R. Fraga, E. F. S. Balau, A. Blanco, R. Ferreira-Marques and A. J. P. L. Policarpo, "Performance of an optical readout GEM-based TPC," Nucl. Instrum. Meth. A 535 (2004) 231. doi:10.1016/j.nima.2004.07.126

[9] M. M. F. R. Fraga, F. A. F. Fraga, S. T. G. Fetal, L. M. S. Margato, R. Ferreira-Marques and A. J. P. L. Policarpo, "The GEM scintillation in He CF-4, Ar CF-4, Ar TEA and Xe TEA mixtures," Nucl. Instrum. Meth. A 504 (2003) 88. doi:10.1016/S0168-9002(03)00758-7

[10] M. Marafini, V. Patera, D. Pinci, A. Sarti, A. Sciubba and E. Spiriti, "ORANGE: A high sensitivity particle tracker based on optically read out GEM,” Nucl. Instrum. Meth. A 845 (2017) 285. doi:10.1016/j.nima.2016.04.014

[11] M. Marafini, V. Patera, D. Pinci, A. Sarti, A. Sciubba and E. Spiriti, "High granularity tracker based on a Triple-GEM optically read by a CMOS-based camera,” JINST 10 (2015) no.12, P12010 doi:10.1088/1748-0221/10/12/P12010 [arXiv:1508.07143 [physics.ins-det]].

[12] R. Veenhof, “GARFIELD, recent developments,” Nucl. Instrum. Meth. A 419 (1998) 726. doi:10.1016/S0168-9002(98)00851-1 